\title{
Évolution des teneurs en éléments minéraux des feuilles de niébé (Vigna unguiculata (L.) Walp.) cultivé dans la zone de Boundji en République du Congo
}

\author{
Joseph YOKA ${ }^{*}$, Jean Joël LOUMET01, Julien Gaudentius DJEGO2, Parisse AKOUANG01, Daniel \\ EPRON ${ }^{3}$ \\ (1) Université Marien Ngouabi, Faculté des Sciences et Techniques, Laboratoire de Botanique et Ecologie, BP: 69, \\ Brazzaville, Congo; Email: joseph yoka@yahoo.fr ; loumeto@hotmail.com ; parakouango@yahoo.fr \\ (2) Université d'Abomey-Calavi, Faculté des Sciences Agronomiques, Laboratoire Ecologie Appliquée. \\ Email: gdgego@yahoo.fr ; \\ (3) Ecologie et Ecophysiologie Forestières (UMR 1137 INRA-UDL), Faculté des Sciences, Université de Lorraine, \\ B.P. 239, 54506 Vandoeuvre-les Nancy, France. Email: daniel.epron@univ-lorraine.fr \\ *Auteur correspondant: Email: joseph_yoka@yahoo.fr
}

Original submitted in on $6^{\text {th }}$ May 2014. Published online at www.m.elewa.org on $31^{\text {st }}$ July 2014. http://dx.doi.org/10.4314/jab.v79i1.1

\section{RÉSUMÉ}

Objectif: Le niébé (Vigna unguiculata (L.) Walp.) est une légumineuse dont les feuilles peuvent être utilisées dans l'alimentation du bétail. L'objectif de l'étude est d'apprécier l'évolution des teneurs en éléments minéraux des feuilles de niébé au cours de son cycle de développent et de déceler la meilleure période de récolte de ce fourrage dans les conditions pédoclimatiques de la zone de Boundji.

Méthodologie et résultats : Les feuilles de niébé ont été prélevées à 1, 2 et 3 mois après le semis. Séchées à l'étuve à $60^{\circ} \mathrm{C}$ jusqu'à poids constant, elles ont subi des analyses chimiques au laboratoire. Les résultats obtenus montrent que les teneurs en divers éléments minéraux évoluent différemment au cours du cycle de développement de la plante.

Conclusion et application des résultats : Dans l'ensemble, les teneurs en éléments majeurs et en oligoéléments peuvent couvrir les besoins de croissance, d'entretien, de reproduction et de production de lait des animaux durant les trois mois du cycle. Cependant la période de 1 mois après le semis semble la plus favorable à la récolte des feuilles.

Mots clés: Niébé, cycle de développement, fourrage, éléments minéraux, zone de Boundji

Evolution of mineral contents of leaves of cowpea (Vigna unguiculata (L.) Walp.) grown in the area Boundji Republic of Congo

Abstract

Objective: Cowpea (Vigna unguiculata (L.) Walp.) is a legume whose leaves can be used in cattle feed . The objective of the study was to assess changes in mineral contents of cowpea leaves during its growing cycle and to identify the best period of harvest in soil and climatic conditions of the Boundji area . 
Methodology and results: Cowpea leaves were collected at 1, 2 and 3 months after sowing. Dried in an oven at $60^{\circ} \mathrm{C}$ to constant weight, they were subjected to chemical analysis in the laboratory. The results show that the levels of various minerals behave differently during the development cycle of the plant. Conclusion and application of results: Overall, the levels of major elements and trace elements can cover the needs of growth, maintenance, reproduction and milk production of animals during three months of the cycle. However the period of one month after sowing seems most favorable to harvest the leaves.

Key words: Cowpea, development cycle, forage, mineral contents, Boundji area

\section{INTRODUCTION}

Le niébé Vigna unguiculata (L.) Walp.) est une légumineuse de régions chaudes d'origine africaine cultivée pour des besoins d'alimentation humaine et de bétail. La production mondiale du niébé s'élève à plus de 5,7 millions de tonnes de graines sèches par an, sur 7,5 millions ha en 2008, dont $70 \%$ sont réalisées en Afrique (Tengo, 2011). La superficie cultivée annuellement dans le monde est estimée à plus de 12,5 millions d'hectares dont environ 9,8 millions d'hectares sont réalisés en Afrique de l'Ouest, faisant de cette région la première productrice et consommatrice de niébé dans le monde (CGIAR 2001). Les principaux pays producteurs en Afrique de l'Ouest sont le Nigéria, le Niger, le Mali, le Burkina Faso, le Sénégal et le Ghana. En Afrique tropicale, les agriculteurs peuvent récolter jusqu'à $400 \mathrm{~kg} / \mathrm{ha}$ de feuilles de niébé. Au Nigéria la moyenne du rendement en graines sèches de quelques cultivars de niébé est de 1,4-1,7 t/ha. La moyenne mondiale en graines sèches de niébé est faible, $240 \mathrm{~kg} / \mathrm{ha}$. Le rendement moyen en graines sèches de niébé dans l'agriculture de subsistance en Afrique tropicale est de $100-500 \mathrm{~kg} / \mathrm{ha}$. Le rendement moyen est de $110 \mathrm{~kg} / \mathrm{ha}$ au Sénégal, $120 \mathrm{~kg} / \mathrm{ha}$ au Niger, $400 \mathrm{~kg} / \mathrm{ha}$ au Nigeria et $900 \mathrm{~kg} / \mathrm{ha}$ aux Etats Unis d'Amérique (Yoka et al., 2014). Au Congo, en Afrique centrale, la culture du niébé est très limitée. Le niébé s'adapte aux conditions climatiques et édaphiques de la plupart des pays d'Afrique et d'ailleurs. Au Congo, précisément dans la Vallée du Niari (sud du pays), les paysans cultivent le niébé pour des besoins de consommation locale. Dans les Plateaux Batékés (Centre du Congo), il est cultivé à des fins expérimentales (Ampion 2009). Dans la Cuvette congolaise (nord du pays) en général et dans la zone de Bopudji en particulier, le niébé n'est également cultivé qu'à des fins expérimentales (Yoka et al., 2014). Dans cette zone, l'élevage bovin extensif se développe, avec comme conséquence la surexploitation des pâturages (Yoka et al., 2011). Dans l'optique de résoudre quantitativement et qualitativement le problème d'affourragement des animaux, des essais de culture de niébé ont été réalisés. Les feuilles récoltées à différents stades du cycle de développement servent de fourrages pour l'alimentation des animaux. Cependant, on connaît très peu de choses sur les teneurs en éléments minéraux des feuilles de niébé cultivées dans cette zone et l'évolution de ces teneurs au cours du cycle de végétation (Yoka et al., 2012). Le dosage des éléments minéraux des fourrages est donc nécessaire, car leurs carences entrainent des troubles graves pour la santé des animaux domestiques (IEMVT, 1988). La carence en cuivre se manifeste par des anémies, des troubles osseux, des ralentissements de croissance, des amaigrissements, des troubles cardiaques et une infécondité. Pour le zinc, elle se manifeste par une inappétence, des dermites, une infécondité due à des troubles de la spermatogenèse chez le taureau et de toutes les phases de reproduction s'étendant de l'œstrus jusqu'au vêlage chez la vache. Des carences en phosphore peuvent se traduire chez la vache par deux ou trois ans sans vêler (Lamand, 1972 ; Conrad et al., 1985). Une carence en calcium retarde la croissance et cause des convulsions tétaniques en cas d'extrême déficience (Conrad et al., 1985). La carence en cobalt provoque un déficit de croissance, une maigreur inexplicable par la quantité et la qualité de la ration, le poil piqué et rugueux sur le garrot. 
Au regard de ces manifestations constatées chez les bovins, il est simple de dire que les éléments minéraux jouent un rôle important dans la vie des animaux. D'où l'intérêt porté à l'analyse de ces éléments minéraux dans les feuilles de niébé cultivé dans la zone de Boundji. En dehors des teneurs en éléments minéraux des feuilles de niébé et leur évolution au cours du développement, la période favorable de récolte est aussi un phénomène mal connu. Ces

\section{MATÉRIEL ET MÉTHODES}

Milieu d'étude : L'étude a été réalisée dans la partie sud-ouest de la Cuvette congolaise, précisément dans la localité de Boundji, située entre $0^{\circ}$ et $2^{\circ}$ de latitude Sud et entre $15^{\circ}$ et $16^{\circ}$ de longitude Est. Le climat de la zone d'étude est de type sub-équatorial (SambaKimbata, 1991). La température moyenne annuelle de la zone d'étude est de $25,5^{\circ} \mathrm{C}$. Les minima moyens sont de $19,9^{\circ} \mathrm{C}$ atteints en juillet et les maxima moyens de $31,9^{\circ} \mathrm{C}$ en mars. La pluviométrie moyenne annuelle de la zone d'étude est de $1657 \mathrm{~mm}$. Les précipitations sont presque permanentes. Les mois d'avril et d'octobre sont les plus pluvieux de l'année dans la Cuvette congolaise. Le maximum de précipitations est enregistré en octobre. II n'y a pas de période écologiquement sèche; seule une diminution de précipitations est notée en juin-juillet-août et en décembre-janvier. L'humidité relative moyenne annuelle de la zone d'étude est toujours élevée (98\%). Les sables Batékés et les alluvions sont des formations géologiques présentes dans la zone d'étude (ORSTOM, 1969; Bouka-Biona et Sounga, 2001). Les sols rencontrés sont principalement des sols ferrallitiques fortement désaturés, et des sols hydromorphes (ORSTOM, 1969). Les sols ferralitiques fortement désaturés sont appauvris et formés sur matériaux sableux ou sablo-faiblement argileux, pauvres en bases et très perméables. Les sols hydromorphes occupent de très vastes étendues dans la partie centrale de la zone d'étude. Ces sols sont dans l'ensemble très sableux (86-96\% de sables), riches en sables fins $(62-73 \%)$, pauvres en matière organique $(1,69-1,88 \%)$, en argiles $(0-8,5 \%)$ et très perméables. Le pH oscille entre 5,2 et 5,9 et le rapport $\mathrm{C} / \mathrm{N}$ entre 13 et 20 (Yoka et al., 2007). La végétation de la zone d'étude est dominée par les forêts et les savanes (UICN, 1990). Les savanes sont de quatre connaissances sont pourtant nécessaires afin d'améliorer l'utilisation des feuilles de niébé et les insérer à dessein dans les plans de rationnement des animaux domestiques. L'objectif de la présente étude est d'apprécier l'évolution des teneurs en éléments minéraux des feuilles de niébé au cours de son cycle de développent et de déceler la période favorable pour la récolte $\mathrm{du}$ fourrage en vue d'améliorer qualitativement l'alimentation des animaux domestiques.

types : savane à Hyparrhenia diplandra Stapf, savane à Trachypogon thollonii Stapf, savane à Andropon schirensis Hochst et savane à Loudetia simplex C.E. Hubbard. Les forêts sont également de plusieurs types ; on y trouve des forêts de terre ferme, des forêts marécageuses et des forêts inondables (UICN, 1990). Les forêts mésophiles caducifoliées sont les plus représentatives dans la zone d'étude.

Caractéristiques botaniques et écologiques du niébé : Le niébé (Vigna unguiculata (L) Walp.) est une plante herbacée annuelle ou vivace, grimpante, rampante ou plus ou moins érigée, cultivée comme annuelle (Photo 1). Sa racine pivotante est bien développée et ses racines latérales et adventives sont nombreuses. La tige du niébé peut atteindre $4 \mathrm{~m}$ de long. Le niébé est originaire d'Afrique, où l'on trouve une grande diversité génétique chez le type sauvage sur tout le continent, l'Afrique australe étant la plus riche (Yoka et al., 2014). Le niébé est le légume sec le plus important des zones de savane d'Afrique occidentale et centrale, où il constitue aussi un important légume vert et une précieuse source de fourrage. II sert de fourrage en Afrique de l'Ouest, en Asie (en Inde surtout) et en Australie ; soit les animaux le broutent directement, soit il est coupé et mélangé à des céréales sèches destinées à l'alimentation du bétail. Aux Etats-Unis et ailleurs, le niébé se cultive comme engrais vert et plante de couverture. La croissance et le développement du niébé peuvent être influencés par plusieurs facteurs parmi lesquels, les besoins en eau, les sols, la température. Les premières études sur l'écologie du niébé ont permis de déterminer les besoins en eau de ce dernier qui sont de $200 \mathrm{~mm}$ pour une production moyenne de l'ordre de $1 \mathrm{t} / \mathrm{ha}$, équivalent à une pluviométrie de 300 à $350 \mathrm{~mm}$ (Freteaud, 1983). 


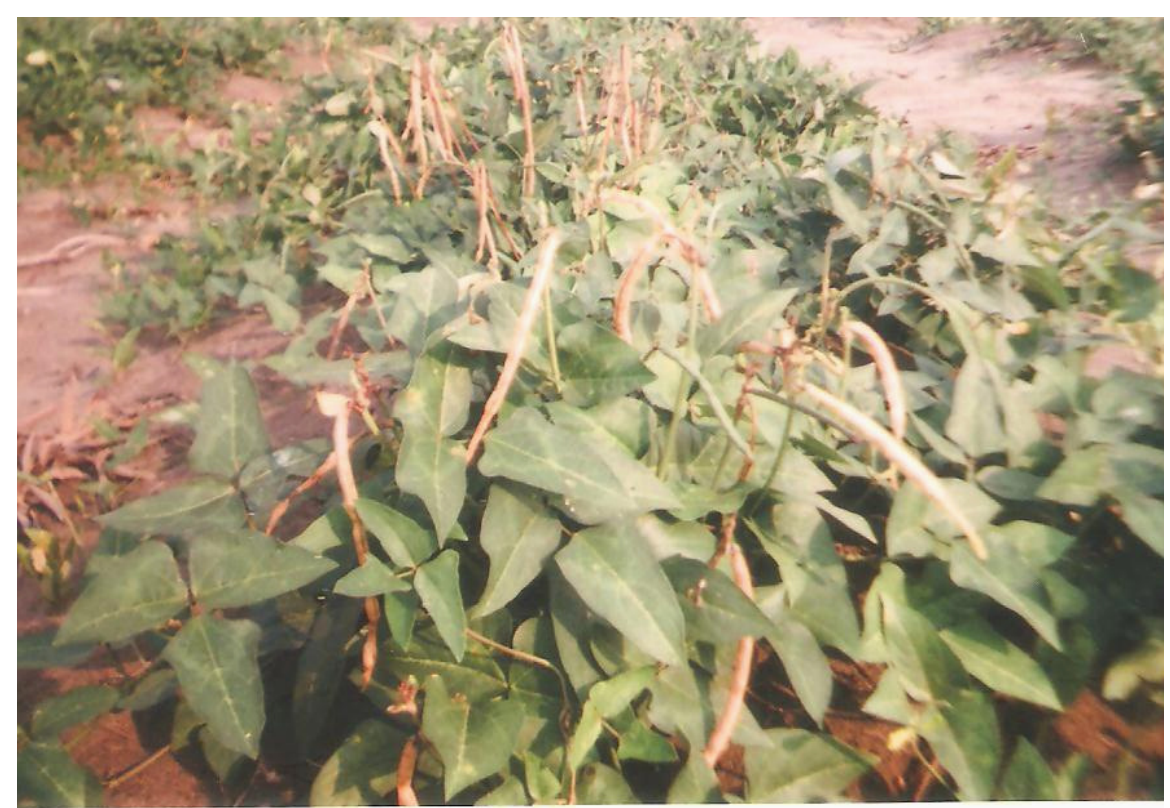

Photo 1: Plants de niébé en gousses, à deux mois après le semis

Le niébé est une légumineuse qui supporte la sécheresse. Selon Goldsworthy et al. (1984), cette plante évite la sècheresse par la réduction des surfaces des folioles, la diminution de la conductance stomatique et le changement dans l'orientation des folioles. Le niébé est sensible à l'engorgement d'eau, car selon ces mêmes auteurs, mêmes de courtes périodes de submersion de son système racinaire peuvent avoir des effets graves sur la production de matières sèches, la fixation symbiotique de l'azote atmosphérique et éventuellement sur le rendement. II convient cependant de noter qu'une forte humidité est néfaste pour le niébé, car elle s'accompagne toujours de dégâts sévères dus aux insectes et à des maladies qui pourraient compromettre la production (Adam, 1986). Le niébé n'est pas exigent du point de vue du sol; il germe bien sur une vaste gamme de sols à condition que ces derniers soient bien drainés. Le rendement optimal en gousses est obtenu sur des sols tourbeux, riches en matière organique. Le niébé est très sensible aux sels, mais tolère l'acidité. Néanmoins le $\mathrm{pH}$ du sol optimal pour la culture du niébé varie entre 6 et 7,5 (Denis, 1984). La température a une grande influence sur le développement du niébé. La température moyenne pendant le cycle végétatif varie entre 25 et $28^{\circ} \mathrm{C}$. La température moyenne de germination se situe entre 15 et $30^{\circ} \mathrm{C}$. Toutefois, les graines peuvent germer à des températures qui varient entre 10 et $40^{\circ} \mathrm{C}$ (Denis, 1984). Selon le même auteur, le niébé supporte des températures assez élevées à condition qu'il y ait une alimentation hydrique suffisante. Le niébé est aussi sensible aux basses températures, car le gel lui est toujours fatal (Craufurd et al., 1997). Le matériel végétal utilisé pour la présente étude est constitué des feuilles d'un cultivar de niébé récoltées à 1,2 et 3 mois après le semis. Ce cultivar est caractérisé par des graines de couleur blanche avec une tache noire dans la partie incurvée. II pourrait provenir de l'Afrique de l'Ouest. Aucune indication n'est donc disponible concernant son origine. Les graines mises à germer ont été achetées au marché, à Brazzaville (Congo), sans indication sur leur provenance.

Dispositif expérimental: En pleine savane à Trachypogon thollonii, une parcelle expérimentale de 0,5 ha a été choisie pour un essai de culture d'un cultivar de niébé retenu dont les graines ont été achetées au marché Total de Brazzaville. Selon les vendeuses, les graines de ce cultivar proviendraient de l'Afrique de l'Ouest. Sa variété n'étant pas maîtrisée, ce cultivar n'a pas un nom particulier; c'est donc un "tout venant". La préparation du champ a été faite manuellement en se servant des houes, machettes et râteaux. Des buttes ont été confectionnées pour le semis. Ces buttes ont en moyenne $4 \mathrm{~m}$ de longueur et $2 \mathrm{~m}$ de largeur. L'espacement entre deux buttes est en moyenne de $1 \mathrm{~m}$. 
Semis du niébé et prélèvement des échantillons de feuilles pour les analyses chimiques : Sur le terrain, le semis a eu lieu en avril 2012 (période pluvieuse) sur des buttes avec un espacement de $75 \mathrm{~cm} \times 50 \mathrm{~cm}$ (Dugje et al., 2009). Le champ a été travaillé manuellement par des femmes paysannes. Trois graines ont été semées par poquet. Le semis a été fait en ligne sur des buttes. Le prélèvement des échantillons de feuilles est intervenu à partir d'un mois après le semis durant tout le cycle végétatif. Ces échantillons ont été prélevés dans des placettes de 1 $\mathrm{m}^{2}$, avec quatre répétitions. Ils ont été séchés d'abord à l'air libre, sur le terrain, puis à l'étuve, au laboratoire, à $60^{\circ} \mathrm{C}$ jusqu'à poids constant. Des échantillons moyens mensuels ont été obtenus et envoyés au laboratoire

\section{RÉSULTATS}

Les apports recommandés en éléments minéraux dans les rations chez les ruminants (Tableau 1) et les teneurs moyennes obtenues après les analyses chimiques sont présentées dans le Tableau 2. Dans l'ensemble, les résultats montrent que les teneurs en éléments minéraux évoluent différemment dans les feuilles de niébé au cours du cycle de développent de la plante qui dure 3 mois. Certains éléments minéraux de la plante augmentent tandis que d'autres diminuent. Aussi bien pour les minéraux majeurs que pour les oligo-éléments, II en existe qui ont tendance à garder la stabilité de leurs teneurs jusqu'à trois mois après le pour des analyses chimiques à l'Institut National de la Recherche Agronomique (INRA), centre de Bordeaux, en France. Le dosage de l'azote et du carbone a été fait par la méthode Dumas (Dumas, 1831). Quant aux autres éléments minéraux à savoir le phosphore, le potassium, le calcium, le magnésium, le sodium, le fer, le manganèse, le zinc et le cuivre totaux, leur dosage a été fait par la méthode ICP-Radial (Masson et al., 1999). Les cendres et la matière organique ont été obtenues par calcination au four à moufle à $480^{\circ} \mathrm{C}$ pendant 5 heures. Les valeurs moyennes des teneurs minérales ont été calculées. Pour le traitement des données, les analyses statistiques faites ne concernent que le calcul de la moyenne et des écarts types.

semis. A titre d'illustration pour les constats sus énumérés, les teneurs moyennes des cendres et l'azote des feuilles de niébé diminuent au cours du cycle végétatif. La diminution est respectivement de 5 et $7 \mathrm{~g} / \mathrm{kg} \mathrm{MS}$ en passant de 1 à 3 mois après le semis. Par contre, les teneurs moyennes en matière organique et en carbone quant à elles augmentent avec l'âge de la plante. Ce qui se justifie par l'accumulation de la biomasse végétale au cours du cycle de développement de la plante. Ces teneurs augmentent de $5 \mathrm{~g} / \mathrm{kg}$ MS pour la matière organique et plus de 50 $\mathrm{g} / \mathrm{kg}$ MS à 3 mois après le semis pour le carbone.

Tableau 1: Apports recommandés de minéraux dans les rations pour ruminants: cas de bovins

\begin{tabular}{|c|c|c|c|c|c|c|c|c|c|}
\hline \multirow[t]{2}{*}{ Recommandation } & \multicolumn{5}{|c|}{ Eléments majeurs (g/kg MS } & \multicolumn{4}{|c|}{ Oligo-éléments (mg/kg MS) } \\
\hline & $\mathbf{P}$ & $\mathrm{Ca}$ & $\mathrm{Mg}$ & $\mathrm{K}$ & $\mathrm{Na}$ & $\mathrm{Cu}$ & $\mathrm{Zn}$ & $\mathrm{Mn}$ & $\mathrm{Fe}$ \\
\hline Bovins en croissance de $200 \mathrm{~kg}$ de PV & - & - & - & - & - & 10 & 50 & 50 & 30 \\
\hline Entretien & 1,2 & 1,8 & - & - & - & - & - & - & - \\
\hline $100 \mathrm{~g} / \mathrm{j}$ de GQM & 1,7 & 2,4 & - & - & - & - & - & - & - \\
\hline Vache reproductrice de $300 \mathrm{~kg}$ de PV & 2,1 & 3,1 & 1,0 & 5,0 & 1,2 & - & - & - & - \\
\hline Taries gestantes & 2,5 & 3,4 & 1,5 & 4,2 & 1,0 & - & - & - & - \\
\hline $7 \mathrm{~kg}$ de lait/j & 3,5 & 6,2 & 1,6 & 6,7 & 1,5 & - & - & - & - \\
\hline Adapté d'après & \multicolumn{5}{|c|}{ Underwood (1981) } & \multicolumn{4}{|c|}{ INRA (1988) } \\
\hline
\end{tabular}

$\mathrm{PV}=$ poids vif; $\mathrm{GQM}=$ gain quotidien moyen; $\mathrm{P}=$ phosphore; $\mathrm{Ca}=$ calcium; $\mathrm{Mg}=$ magnésium; $\mathrm{K}=$ potassium; $\mathrm{Na}=$ sodium; $\mathrm{Cu}=$ cuivre; $\mathrm{Zn}=$ zinc; $\mathrm{Mn}=$ manganèse; $\mathrm{Fe}=$ fer.

Tout comme pour l'azote, les éléments minéraux majeurs (phosphore, potassium et le sodium connaissent également une baisse de leurs teneurs moyennes au fur et à mesure de l'accroissement de la plante. La diminution est beaucoup plus importante pour le potassium à 3 mois après le semis que pour le phosphore et le sodium (18 $\mathrm{g} / \mathrm{kg}$ MS contre 0,56 et 0 , $09 \mathrm{~g} / \mathrm{kg}$ MS respectivement) . Le calcium au contraire à tendance à augmenter au cours du cycle végétatif de la plante par rapport aux autres éléments minéraux majeurs. Au regard des résultats rapportés par les analyses de laboratoire, les teneurs en magnésium ont 
tendance à se stabiliser dans les feuilles de niébé jusqu'à 3 mois après le semis. S'agissant des oligoéléments, le fer et le zinc présentent des teneurs moyennes en diminution au cours du cycle de développement. Jusqu'à 3 mois, après le semis, on peut espérer une stabilité des teneurs en manganèse et en cuivre au niveau des feuilles de niébé.

Tableau 2: Teneurs en cendres, en matière organique et en éléments minéraux des feuilles de niébé prélevées à 1 , 2 et 3 mois après le semis.

\begin{tabular}{|l|l|l|l|}
\hline Eléments analysés & 1 mois après le semis & 2 mois après le semis & 3 mois après le semis \\
\hline Cendres $(\% \mathrm{MS})$ & $16,2 \pm 0,32$ & $11,6 \pm 0,23$ & $10,6 \pm 0,21$ \\
\hline Matière organique $(\% \mathrm{MS})$ & $83,8 \pm 1,7$ & $88,4 \pm 1,8$ & $89,4 \pm 1,8$ \\
\hline Azote $(\mathrm{g} / \mathrm{kg} \mathrm{MS})$ & $35,1 \pm 2,1$ & $34,5 \pm 2,1$ & $28,0 \pm 1,7$ \\
\hline Carbone $(\mathrm{g} / \mathrm{kg} \mathrm{MS})$ & $429 \pm 21$ & $457 \pm 2,1$ & $480 \pm 24$ \\
\hline Phosphore $(\mathrm{g} / \mathrm{kg} \mathrm{MS})$ & $4,75 \pm 0,24$ & $4,07 \pm 0,20$ & $3,19 \pm 0,16$ \\
\hline Potassium $(\mathrm{g} / \mathrm{kg} \mathrm{MS})$ & $25,4 \pm 2,5$ & $16,8 \pm 1,7$ & $7,05 \pm 0,71$ \\
\hline Calcium $(\mathrm{g} / \mathrm{kg} \mathrm{MS})$ & $21,1 \pm 2,1$ & $22,0 \pm 2,2$ & $25,4 \pm 2,5$ \\
\hline Magnésium $(\mathrm{g} / \mathrm{kg} \mathrm{MS})$ & $5,95 \pm 0,59$ & $5,09 \pm 0,51$ & $5,85 \pm 0,59$ \\
\hline Sodium $(\mathrm{g} / \mathrm{kg} \mathrm{MS})$ & 0,113 & 0,0686 & 0,0279 \\
\hline Fer $(\mathrm{mg} / \mathrm{kg} \mathrm{MS})$ & $478 \pm 72$ & $213 \pm 32$ & $197 \pm 30$ \\
\hline Manganèse $(\mathrm{mg} / \mathrm{kg} \mathrm{MS})$ & $188 \pm 19$ & $243 \pm 24$ & $197 \pm 20$ \\
\hline Zinc $(\mathrm{mg} / \mathrm{kg} \mathrm{MS)}$ & $54,2 \pm 5,4$ & $44,1 \pm 4,4$ & $19,9 \pm 2,0$ \\
\hline Cuivre $(\mathrm{mg} / \mathrm{kg} \mathrm{MS})$ & $12,2 \pm 2,4$ & $13,5 \pm 2,7$ & $11,6 \pm 2,3$ \\
\hline
\end{tabular}

Remarquons à travers cette étude que les feuilles de niébé cultivé dans la zone de Boundji présentent des teneurs très élevées en azote., Les teneurs en éléments minéraux majeurs (calcium, potassium phosphore et magnésium) sont largement supérieures aux normes recommandés dans les rations pour ruminants. Par contre, ces feuilles sont très pauvres en sodium et les teneurs enregistrées ne donnent par satisfaction par rapport à la couverture des besoins des ruminants. Concernant les oligo-éléments, les teneurs

\section{DISCUSSION}

Au fur et à mesure de l'accroissement de la plante de niébé, les teneurs en azote et en phosphore des feuilles diminuent. Cette tendance corrobore les résultats de Yoka (2009) qui montrent que les teneurs de ces deux éléments diminuent avec l'âge des espèces herbacées des savanes de la Cuvette congolaise. Cependant, les teneurs en azote (28$35,1 \mathrm{~g} / \mathrm{kg} \mathrm{MS}$ ) des feuilles de niébé sont très élevées par rapport à celles de Hyparrhenia diplandra (10,5$15,5 \mathrm{~g} / \mathrm{kg}$ MS) et de Loudetia simplex (7,1-17,2 $\mathrm{g} / \mathrm{kg}$ MS), deux graminées dominantes des savanes de la Cuvette congolaise. Les teneurs en phosphore des feuilles de niébé sont également élevées par rapport à celles de Hyparrhenia diplandra (3,19-5,75 g/kg MS) et de Loudetia simplex (1,6-2,1 g/kg MS). Les espèces de en fer et en manganèse sont largement supérieures aux normes recommandées quel que soit le stade de développement de la plante. Les teneurs en cuivre suivent la même tendance. Au regard des apports recommandés, les teneurs en zinc des feuilles de niébé ne sont plus satisfaisantes après le premier mois de semis dans la région de Boundji. Dans ce cas, une complémentation alimentaire s'avère nécessaire afin de satisfaire les besoins d'entretien et de croissance des ruminants.

savanes prises globalement ont des teneurs en azote et en phosphore inférieures à celles des feuilles de niébé. Dans les espèces des savanes à Hyparrhenia diplandra, ces teneurs oscillent entre 10,5 et 27,8 g/kg MS, pour l'azote et entre 2,1 et 3,1 $\mathrm{g} / \mathrm{kg}$ MS, pour le phosphore. Dans les espèces des savanes à Loudetia simplex, la tendance est la même, car les teneurs oscillent entre 7,6 et 22,1 $\mathrm{g} / \mathrm{kg} \mathrm{MS}$, pour l'azote, et sont autour de $1,9 \mathrm{~g} / \mathrm{kg} \mathrm{MS}$, pour le phosphore. A partir de ces résultats, nous pouvons dire que la pauvreté en azote et en phosphore des espèces herbacées de savanes qui constituent le fourrage potentiel pour l'alimentation du bétail, pourrait être compensée par les feuilles de niébé. Les teneurs de ces deux éléments minéraux qui malgré leur diminution dans les feuilles de 
niébé au cours du cycle de végétation, restent à un niveau satisfaisant pour couvrir les besoins alimentaires des herbivores domestiques. S'agissant des autres éléments minéraux, les teneurs des feuilles de niébé en éléments majeurs et en oligo-éléments, à 2 mois après le semis, sont élevées par rapport à celles que Diamouangana (2000) et Yoka (2009) ont trouvées respectivement dans les plantes herbacées des savanes de la Vallée du Niari et de la Cuvette congolaise. Selon Diamouangana (2000), Les teneurs en calcium, magnésium et potassium dans les repousses de Hyparrhenia diplandra de 70 jours au ranch de Dihessé (Vallée du Niari) sont respectivement de $3,3 \mathrm{~g} / \mathrm{kg} ; 2,1 \mathrm{~g} / \mathrm{kg}$ et $11,8 \mathrm{~g} / \mathrm{kg}$. Dans la Cuvette congolaise, ces teneurs dans les repousses de Hyparrhenia diplandra de deux mois après le feu, oscillent de 1,96-7,28 g/kg pour le calcium; 1,42-2,70 $\mathrm{g} / \mathrm{kg}$ pour le magnésium et 5,57-11,6 g/kg pour le potassium, selon les stations (Diamouangana, 2000). Elles sont dans l'ensemble proches de celles trouvées par Diamouangana (2000) dans la Vallée du Niari. Dans les mêmes repousses de Hyparrhenia diplandra de 70 jours au ranch de Dihessé, l'auteur a trouvé les teneurs en oligo-éléments ci-après : $15,0 \mathrm{mg} / \mathrm{kg}$ pour le zinc ; $164,4 \mathrm{mg} / \mathrm{kg}$ pour le manganèse et $142,7 \mathrm{mg} / \mathrm{kg}$ pour le fer. Dans la Cuvette congolaise, les teneurs en ces oligo-éléments dans les repousses de 2 mois de Hyparrhenia diplandra, oscillent de 12,5-20,4 mg/kg pour le zinc ; 83,9-121 mg/kg pour le manganèse et $160-657 \mathrm{mg} / \mathrm{kg}$ pour le fer. Le constat est que les teneurs en zinc dans Hyparrhenia récolté dans les deux zones écologiques sont presque identiques. Cependant, la teneur en manganèse est plus élevée à

\section{CONCLUSION}

L'étude a permis de montrer que les teneurs en éléments minéraux des feuilles de niébé évoluent différemment au cours du cycle de végétation de la plante. Les teneurs en azote, en phosphore et en potassium diminuent avec l'âge de la plante, contrairement aux teneurs en calcium qui augmentent. Seules les teneurs en sodium sont entre 1 et 3 mois après le semis. Dans l'ensemble, malgré les différences dans leur évolution, les teneurs en éléments majeurs et en oligo-éléments des feuilles de niébé peuvent couvrir les besoins de croissance, d'entretien, de reproduction et de production de lait des animaux, durant tout le cycle de végétation. La période de 1 mois après le la Dihessé que dans la Cuvette congolaise. Quant à la teneur en fer, elle atteint une valeur très élevée dans la Cuvette congolaise. En tenant compte des seuils critiques, nous pensons que Hyparrhenia diplandra est une graminée fourragère riche en magnésium, potassium, manganèse et fer, et pauvre en calcium, phosphore et zinc, dans les deux zones écologiques (Vallée du Niari et Cuvette congolaise). Ces résultats confortent les observations de Mandiki et al. (1986) dans la sous-région de l'Ituri (République Démocratique du Congo) et celles de Villecourt et al. (1979). Malgré cette richesse de Hyparrhenia diplandra, qui est l'une des espèces dominantes des savanes de la Cuvette congolaise, en certains éléments minéraux, il est nécessaire d'enrichir les pâturages de cette zone par la culture de niébé. Python et Boessinger (2012) ont montré que l'altitude agit sur les minéraux majeurs, le manganèse et le zinc. L'année exerce une influence significative sur une grande partie des teneurs minérales, à l'exception de l'azote et du potassium. Ce qui revient à dire que les teneurs minérales des fourrages varient d'une zone écologique à une autre et d'une année à une autre. Yoka et al. ( 2014) ont montré que le niébé s'adapte bien aux conditions pédoclimatiques de la zone de Boundji dans la Cuvette congolaise. Bossuet et Vadez 2013) ont montré que les légumineuses peuvent améliorer significativement l'alimentation minérale et donc jouer un rôle important dans le développement de l'élevage. La culture de niébé est donc à encourager dans les programmes d'amélioration des pâturages, en vue de fournir le fourrage de qualité aux herbivores domestiques.

semis semble la plus favorable à la récolte des feuilles de niébé pour l'affourragement, car à cette période la plupart des éléments minéraux ont des teneurs élevées. Les feuilles du cultivar de niébé étudié peuvent donc être valorisées dans l'alimentation du bétail, en vue l'amélioration de la productivité des animaux d'élevage. L'évolution des teneurs en éléments minéraux des feuilles d'autres cultivars de niébé feront l'objet de nos prochaines études dans la Cuvette congolaise; ce qui permettra de valoriser la culture de niébé dans les programmes d'amélioration des pâturages. 
RÉFÉRENCES BIBLIOGRAPHIQUES

Adam T, 1986. Contribution à la connaissance des maladies du niébé au Niger avec mention spéciale au Macrophomina phaseolina. Thèse de Docteur Ingénieur en Sciences Agronomiques, Université de Rennes, $128 \mathrm{p}$.

Ampion TL, 2009. Valorisation de quelques variétés de niébé (Vigna unguiculata (L) Walp.) dans l'alimentation animale dans les conditions pédoclimatiques de Nkouo. Mémoire de Diplôme d'Etudes Approfondies, Université Marien Ngouabi, Brazzaville, $54 \mathrm{p}$.

Bossuet J et Vadez V, 2013. S'appuyer sur les multiples bénéfices des légumineuses à graines pour une agriculture plus productive et nutritive dans les tropiques semi-arides. Sécheresse, 24, 4, 314-321.

Bouka-Biona C et Sounga JD, 2001. Corrélation entre la localisation des foyers des séismes et les zones de limitation des horts et grabens du soubassement de la Cuvette congolaise (Afrique centrale). Annales de l'Université Marien Ngouabi, 2 (1), 125-139.

CGIAR, 2001. Cowpea (Vigna unguiculata). CGIAR on line/CGIAR Research: Areas of Research, $<$ http:/www.cgiar.org/research/res_cowpea.ht $\mathrm{ml}>$, Washington, D.C.

Conrad JH, Mcdowell LR, Ellis GL, Loosli JK, 1985. Minéraux pour les ruminants des pâturages des régions tropicales. USAID, Univ. of Florida, Garnisville, $96 \mathrm{p}$.

Craufurd PQ, Ellis RH, Summerfield RJ, Menin L, 1997. Photoperiod, temperature and growth and developpement of cowpea. Vigna unguiculata in Advances in cowpea reseach, 75-86.

Denis JJ, 1984. Manuel de principales cultures sahéliennes. Tome 2, $234 \mathrm{p}$.

Diamouangana J, 2000. Teneurs en éléments minéraux des fourrages de la plaine de Dihessé (CongoBrazzaville) : proposition de complémentation pour bovins. Annales de l'Université Marien Ngouabi, 1, 103-115.

Dugje IY, Omoigui LO, Ekeleme F, Kamara AY, Ajeigbe $H, 2009$. Production du niébé en Afrique de I'Ouest: Guide du paysan. Document de I'IITA, $20 \mathrm{p}$.

Dumas JBA, 1831. Procédés de l'analyse organique. Annal. Chem. Phys., Paris, 47 (2), 198-213.
Freteaud, 1983. Synthèse des recherches agro climatologiques sur le niebe. CNRA, Bambey, $44 \mathrm{p}$.

Goldsworthy PR and Fisher NM, 1984. The physiology of tropical Field crops Wiley. Inter science publication John Wiley and sons, $664 \mathrm{p}$.

IEMVT, 1988. Manuel vétérinaire des agents techniques de l'élevage tropical. Ministère de la Coopération et du développement, la documentation française, Paris, $533 \mathrm{p}$.

INRA, 1988. Principe de la nutrition et de l'alimentation des ruminants. INRA, Versailles, France, 596 $p$.

Lamand M, 1972. Causes, conséquences biochimiques, pathologiques et prophylaxie des carences en oligo-éléments chez les ruminants. Aliments, vie, 59 (2), 154-172.

Mandiki SNM, Kiatoto, Olenga L, 1986. Composition minérale des fourrages de la Sous-région de I'Ituri (Zaïre) et proposition de complémentation pour bovins. Revue Elev. Med. Vet. Pays trop., 39 (4), 425-434.

Masson P, Orignac D, Vives A, Prunet T, 1999. Matrix effects during trace element analysis in plant samples by inductively coupled plasma atomic emission spectrometry with axial view configuration and ultrasonic nebulizer. Analusis, 27, 813-820.

ORSTOM, 1969. Atlas du Congo, 10 cartes couleur avec notice, ORSTOM, Brazzaville.

Python $P$ et Boessinger M, 2012. Facteurs d'influence sur les valeurs nutritives des fourrages secs. Recherche Agronomique Suisse, 3, 1, 36-43.

Samba-Kimbata MJ, 1991. Précipitations et bilan de l'eau dans le bassin forestier du Congo et ses marges. Thèse d'Etat, Centre de Recherche de Climatologie, Dijon, 241p. + 163 fig.

Tengo NS, 2011. Technique de conservation des légumineuses et sécurisation de la production des paysans: cas du niébé dans le département du Diamare. Mémoire de Master, Institut Supérieur de Sahel, Université de Maroua, $142 \mathrm{p}$.

UICN, 1990. La conservation des écosystèmes forestiers du Congo. UICN, Brazzaville, $187 \mathrm{p}$.

Underwood CJ, 1981. The mineral nutrition of livestock. 2nd ed. Farnham Royal, Slough (U.K). CAB, $180 \mathrm{p}$. 
Villecourt P, Scmidt W, Cesar J, 1979. Recherche sur la composition chimique $(\mathrm{N}, \mathrm{P}, \mathrm{K})$ de la strate herbacée de la savane de Lamto (Côte d'ivoire). Rév. Ecol. Biol. Sol, 16 (1) : 9-15.

Yoka J, 2009. Contribution à l'étude phyto-écologique et des potentialités fourragères des savanes de la Cuvette congolaise (République du Congo). Thèse de Doctorat, Université Marien Ngouabi, Brazzaville, $137 \mathrm{p}$.

Yoka J, Loumeto JJ, Vouidibio J, 2007. Quelques caractéristiques écologiques des savanes de la zone d'Ollombo (Cuvette congolaise, République du Congo). Annales de l'Université Marien Ngouabi , 8 (4): 75-87.

Yoka J, Amiaud B, Epron D, Loumeto JJ, Vouidibio J, 2011. Evolution sous pâture de la composition floristique des savanes de la Cuvette congolaise (République du Congo). Annales de l'Université Marien Ngouabi, 12 (4): 23-38.

Yoka J, Loumeto JJ, Vouidibio J, Epron D, 2012. Evaluation du profil minéral des espèces fourragères et de la capacité de charge des savanes de la Cuvette congolaise (République du Congo). Annales des Sciences Agronomiques, 16 (2): 125-141.

Yoka J, Loumeto JJ, Djego JG, Houinato M, Aouango $P, 2014$. Adaptation d'un cultivar de niébé (Vigna unguiculata L. (Walp.) aux conditions pédoclimatique de la zone de Boundji (République du Congo). Afrique Science, 10 (1): 217-225. 